\title{
Efeitos da desnervação intrínseca do jejuno após enterectomia extensa na síndrome do intestino curto em ratos ${ }^{1}$
}

\author{
Effects of intrinsic denervation of the jejunum after extensive enterectomy in the rat \\ bowel syndrome
}

\begin{abstract}
Carlos Eduardo Verdiani de Carvalho, ${ }^{2}$ Filipe Volpe D'Angieri Basile ${ }^{2}$, Marcelo Vinícius Oliveira Vespúcio ${ }^{3}$, Antonio Carlos Garrido Iglesiass ${ }^{4}$, Nelson Fabrício Gava ${ }^{5}$, Sérgio Britto Garcia ${ }^{6}$
\end{abstract}

1. Trabalho realizado no Departamento de Patologia da Faculdade de Medicina de Ribeirão Preto -USP.

2. Médico Residente do Hospital das Clínicas da Faculdade de Medicina de Ribeirão Preto -USP.

3. Cirurgião Dentista, Doutorando em Patologia na Faculdade de Medicina de Ribeirão Preto -USP.

4. Professor Titular do Departamento de Cirurgia da UNIRIO

5. Aluno de Graduação da Faculdade de Medicina de Ribeirão Preto -USP.

6. Professor Doutor do Depto. de Patologia da Faculdade de Medicina de Ribeirão Preto -USP.

\section{RESUMO}

Objetivo: Investigar em ratos Wistar as respostas adaptativas da mucosa em conseqüência da desnervação intrínseca do jejuno após ressecção intestinal extensa. Métodos: Utilizaram-se 30 ratos distribuídos em três grupos segundo o procedimento realizado: C (controle), R (ressecção intestinal) e D (ressecção intestinal e desnervação intrínseca do jejuno). Posteriormente foi avaliado o ganho de peso e realizado estudos morfométrico da mucosa intestinal. Resultados: Os animais do grupo D apresentaram ganho ponderal consideravelmente maior do que os do grupo $R$ ( $D=312,2 \pm 21 \mathrm{~g} e$ $\mathrm{R}=196,7 \pm 36,2 \mathrm{~g})$. A contagem neuronal mostrou diminuição na população de neurônios mientéricos no grupo $\mathrm{D}(344,8 \pm 34,8$ neurônios/mm de jejuno) em relação aos outros grupos $(\mathrm{R}=909,0 \pm 55,5$ e $\mathrm{C}=898,5 \pm 73,3)$. A área do epitélio da mucosa jejunal foi maior no grupo $\mathrm{D}\left(10,8 \pm 4,3 \mathrm{~mm}^{2}\right)$ em comparação aos grupos $\mathrm{R}\left(7,3 \pm 3,9 \mathrm{~mm}^{2}\right)$ e $\mathrm{C}\left(5,8 \pm 3,0 \mathrm{~mm}^{2}\right)$. O índice de proliferação celular epitelial da mucosa foi maior no grupo D (48,7\%), em relação aos grupos R (31,9\%) e C (23,6\%). Conclusões: O modelo experimental mostrou-se eficaz em melhorar o ganho ponderal dos animais submetidos à ressecção intestinal extensa, provocando intensificação da resposta hiperplásica da mucosa, a qual provavelmente levou a aumento da superfície de absorção de nutrientes. Abrem-se boas perspectivas para novas abordagens cirúrgicas para a síndrome do intestino curto.

Descritores: Síndrome do Intestino Curto. Compostos de Benzalcônio. Ratos Wistar. Jejuno.

\begin{abstract}
Purpose: To investigate the effects of intrinsic denervation of the jejunum after the extensive intestinal resection in rats. Methods: Thirty male Wistar rats were distributed into three groups, depending on the experimental procedure: Group C (control), Group R (resection) and Group D (resection plus denervation). The body weight gain and a histomorphometric study of the jejunal mucosa were performed. Results: The mean body weight of the group D animals showed a higher increase when compared to group $\mathrm{R}(\mathrm{D}=312,2 \pm 21 \mathrm{~g}$ and $\mathrm{R}=196,7 \pm 36,2 \mathrm{~g})$. The number of jejunum myenteric neurons was smaller in group $\mathrm{D}(344,8 \pm 34,8$ neurons $/ \mathrm{mm})$ when compared to other groups $(\mathrm{R}=909,0 \pm 55,5$ and $\mathrm{C}=898,5 \pm 73,3)$. A hyperplasia of the jejunum mucosal epithelium was observed in the group $\mathrm{D}$ but also in the group $\mathrm{R}\left(\mathrm{R}=7,3 \pm 3,9 \mathrm{~mm}^{2}\right.$ and $\left.\mathrm{D}=10,8 \pm 4,3 \mathrm{~mm}^{2}\right)$, when compared to group $\mathrm{C}\left(\mathrm{C}=5,8 \pm 3,0 \mathrm{~mm}^{2}\right)$. The epithelial cell proliferation of the jejunum was higher in group $\mathrm{D}$ animals $(48,7 \%)$ when compared to the other groups $(\mathrm{R}=31,9 \%$ and $\mathrm{C}=23,6 \%)$. Conclusions: The denervated animals presented an increase the body weight gain and mucosal cell proliferation responses when compared to the control group. This experimental model may provide new strategies for the surgical treatment of the short bowel syndrome.
\end{abstract}

Key words: Short Bowel Syndrome. Benzalkonium Compounds. Rats, Wistar. Jejunum.

\section{Introdução}

A Síndrome do intestino curto (SIC) é um conjunto de sinais e sintomas decorrente de um comprimento intestinal insuficiente que pode ser desenvolvido após ressecção de grandes extensões do intestino delgado. As principais adaptações intestinais à ressecção consistem em aumento da superfície absortiva, por aumento no comprimento e calibre da víscera ${ }^{1-3}$. Estas alterações ocorrem com aumento da taxa proliferativa e hiperplasia das camadas musculares do intestino delgado ${ }^{4}$. Apesar dos esforços adaptativos do organismo, a desnutrição e perda de peso são inexoráveis quando a ressecção atinge grandes extensões ${ }^{5}$. As técnicas cirúrgicas existentes não produzem resultados satisfatórios, e o tratamento paliativo consiste em evitar a desnutrição e desidratação através da administração parenteral de soluções hiperosmóticas. Este tratamento além de dispendioso é desconfortável para o paciente ${ }^{5-7}$. 
Experimentalmente, Sawchuk e cols. ${ }^{8}$ demonstraram que a desnervação química por cloreto de benzalcônio (CB) do segmento intestinal remanescente a ressecção aumenta a sobrevida de ratos com SIC. Foi depois observado que a desnervação ileal por CB acarreta dilatação da alça intestinal formando megaileo com aumento da superfície absortiva, permitindo uma melhora nas condições pós-cirurgicas e ganho de peso em ratos com $\mathrm{SIC}^{9}$. Entretanto, não se conhecem os efeitos da desnervação jejunal na SIC. É possível que sejam diferentes daqueles observados após a desnervação ileal, uma vez que Senior e cols. ${ }^{10}$ demonstraram que após ressecção de $70 \%$ do intestino delgado, a resposta celular proliferativa da mucosa jejunal foi muito mais intensa do que a ileal. Baseado nestes fatos, o presente estudo pretende estudar quais as possíveis respostas adaptativas da mucosa no caso de se proceder a desnervação intrínseca do jejuno após ressecção intestinal extensa.

\section{Métodos}

Foram utilizados trinta (30) ratos, da linhagem Wistar, machos, pesando $200 \mathrm{~g}$ no início do experimento, provenientes do Biotério Central da Faculdade de Medicina de Ribeirão Preto - USP. Os animais foram divididos em três grupos: C (controle), R (ressecção intestinal) e D (ressecção intestinal e desnervação). Para a indução da síndrome do intestino curto nos grupos experimentais $\mathrm{R}$ e D, os animais foram submetidos à laparotomia mediana, exposição do intestino delgado do animal, ligadura dos vasos da região a ser ressecada e ressecção de $80 \%$ deste segmento intestinal, preservando o jejuno proximal e o íleo distal. Em seguida fez-se anastomose boca a boca entre a porção remanescente do jejuno e a porção ileal próxima à junção ileocecal com conseqüente aplicação tópica nos cinco $\mathrm{cm}$ proximais do local da anastomose com gaze embebida em solução salina (grupo R) ou cloreto de benzalcônio, solução $2 \mathrm{mM}$ (grupo D) durante 30 minutos. Feito isso, o segmento exposto da cavidade abdominal foi lavado com solução salina $0,9 \%$, as vísceras foram recolocadas e a parede abdominal reconstruída através de sutura em dois planos. $\mathrm{O}$ animais do grupo $\mathrm{C}$ foram submetidos apenas a laparotomia e sutura. Para avaliação indireta do estado nutricional, os pesos dos animais foram verificados na época da cirurgia e ao final do experimento. Após 90 dias do procedimento cirúrgico, os animais foram sacrificados e o segmento intestinal restante foi coletado e fixado em em formol (10\%) para estudo imunoistoquímico e histológico com coloração de H\&E.

\section{Estudo morfométrico e morfocinético do epitélio da mucosa}

O estudo morfométrico foi realizado através do sistema semi-automático de análise de imagens (MINI-MOP, Kontron â). Os parâmetros analisados foram: a) diâmetro médio da luz dos segmentos jejunais remanescentes à ressecção e do segmento equivalente nos animais do grupo controle, b) área das camadas muscular e mucosa, expressa em $\mathrm{mm}^{2}$ por segmento longitudinal de $1 \mathrm{~cm}$ de comprimento de alça intestinal. Já o estudo morfocinético foi realizado através da contagem imunoistoquímica de marcação da proliferação celular (PCNA). Vinte criptas jejunais foram contadas por animal.

\section{Estudo da população de neurônios mientéricos}

Para confirmar a eficácia da aplicação do CB em induzir desnervação intrínseca da parede jejunal, a população de neurônios mientéricos desta parede foi estimada através de contagem neuronal ${ }^{11}$. Os resultados foram expressos como número de neurônios por milímetro intestinal.

\section{Análise estatística}

A análise dos resultados foi realizada através do teste não paramétrico de Mann Whitney com nível de significância $\mathrm{p}<0,01$.

\section{Resultados}

Nos animais dos grupos $\mathrm{C}$ e $\mathrm{D}$ que sobreviveram até o final do período experimental, observou-se boa evolução do ganho ponderal, sendo que na época do sacrifício os animais do grupo D apresentaram peso corporal consideravelmente maior do que os do grupo R (Tabela 1).

TABELA 1 - Peso corporal e ganho ponderal dos animais durante o período experimental.

\begin{tabular}{l|l|l|l}
\hline Grupo & $\begin{array}{l}\text { Peso corporal no } \\
\text { início do período } \\
\text { experimental (g) }\end{array}$ & $\begin{array}{l}\text { Peso corporal no } \\
\text { final do período } \\
\text { experimental (g) }\end{array}$ & $\begin{array}{l}\text { Ganho } \\
\text { ponderal } \\
\text { médio (g) }\end{array}$ \\
\hline $\mathrm{D}$ & $158,3 \pm 12,1$ & $470,5 \pm 33,1 * *$ & $312,2 \pm 21$ \\
\hline $\mathrm{R}$ & $149,9 \pm 13,0$ & $346,6 \pm 49,2 *$ & $196,7 \pm 36,2$ \\
\hline $\mathrm{C}$ & $150,9 \pm 11,8$ & $598,3 \pm 32,3$ & $447,4 \pm 20,5$ \\
\hline
\end{tabular}

** $O$ teste estatístico de Mann-Whitney resultou em $\mathrm{p}<0.01$ (estatisticamente significativo), quando comparado aos demais grupos. * O teste estatístico de Mann-Whitney resultou em $\mathrm{p}<0.01$ (estatisticamente significativo), quando comparado ao grupo controle (C).

A contagem neuronal confirmou a existência da diminuição da população de neurônios mientéricos nos animais do grupo D, quando comparados aos grupos C e R (Tabela 2). A análise histopatológica dos cortes de jejuno mostrou que a ressecção intestinal e a desnervação pelo CB causaram alterações hiperplásicas na mucosa jejunal, mais acentuadas na última situação. Estes achados foram confirmados pela análise morfométrica, a qual mostrou um aumento de área da mucosa da ordem de 50\% nos animais que sofreram ressecção intestinal e de $100 \%$ naqueles também desnervados (Tabela 3).

TABELA 2 - População neuronal do plexo mientérico jejunal, expressa em número de neurônios $/ \mathrm{mm}$ de comprimento intestinal.

\begin{tabular}{cc}
\hline Grupo & Neurônios mientéricos/mm de jejuno \\
\hline D & $344,8 \pm 34,8^{*}$ \\
R & $909,0 \pm 55,5$ \\
C & $898,5 \pm 73,3$
\end{tabular}

*O teste estatístico de Mann-Whitney resultou em $\mathrm{p}<0.01$ (estatisticamente significativo), quando comparado aos demais grupos. 
TABELA 3 - Área média das camadas muscular e mucosa por mm de extensão do jejuno e diâmetro médio da alça jejunal nos grupos $\mathrm{D}, \mathrm{R}$ e C.

\begin{tabular}{l|l|l|l}
\hline Grupo & $\begin{array}{l}\text { Área da camada } \\
\text { muscular }\left(\mathbf{m m}^{2}\right)\end{array}$ & $\begin{array}{l}\text { Área da camada } \\
\text { mucosa }\left(\mathbf{m m}^{2}\right)\end{array}$ & $\begin{array}{l}\text { Diâmetro médio } \\
\text { do jejuno }(\mathbf{m m})\end{array}$ \\
\hline $\mathrm{D}$ & $4,3 \pm 1,8 *$ & $10,8 \pm 4,3^{*}$ & $12,1 \pm 1,1 *$ \\
$\mathrm{R}$ & $2,2 \pm 0,8$ & $7,3 \pm 3,9$ & $0,9 \pm 0,2$ \\
$\mathrm{C}$ & $1,5 \pm 0,7$ & $5,8 \pm 3,0$ & $0,9 \pm 0,1$ \\
\hline
\end{tabular}

*O teste estatístico de Mann-Whitney resultou em $\mathrm{p}<0.01$ (estatisticamente significativo), quando comparado aos demais grupos.

Houve também um aumento estatisticamente significativo, no índice de células marcadas positivamente para o PCNA, indicando aumento da taxa de proliferação celular epitelial da mucosa nos animais do grupo R, com aumento mais pronunciado no grupo D (Tabela 4).

TABELA 4 - Índice de células marcadas positivamente para o PCNA no epitélio da mucosa jejunal nos animais dos $\mathrm{D}, \mathrm{R}$ e $\mathrm{C}$.

\begin{tabular}{cc}
\hline Grupo & Índice de células marcadas pelo PCNA (\%) \\
\hline $\mathrm{D}$ & $48,7^{* *}$ \\
$\mathrm{R}$ & $31,9^{*}$ \\
$\mathrm{C}$ & 23,6 \\
\hline
\end{tabular}

** $\mathrm{O}$ teste estatístico de Mann-Whitney resultou em $\mathrm{p}<0.01$ (estatisticamente significativo), quando comparado aos demais grupos. * O teste estatístico de Mann-Whitney resultou em $\mathrm{p}<0.01$ (estatisticamente significativo), quando comparado ao grupo controle (C).

\section{Discussão}

No presente experimento observamos que a desnervação mientérica do jejuno pelo $\mathrm{CB}$ associada à ressecção intestinal extensa acarreta melhora do ganho ponderal dos animais, uma vez que ao final do período experimental os animais do grupo D apresentaram peso corporal significativamente maior do que aqueles do grupo R. Entretanto observamos que o peso corporal médio no grupo D foi inferior ao do grupo controle, evidenciando que a desnervação mientérica, ainda que tenha melhorado as condições de ganho de peso dos animais não foi suficiente para propiciar reversão completa do quadro de síndrome do intestino curto. Este resultado ficou aquém daquele que observamos em trabalho anterior quando desnervamos o íleo ao invés do jejuno em associação à ressecção intestinal extensa ${ }^{9}$. Não temos uma explicação definitiva sobre este aspecto, mas é possível que a resposta adaptativa do jejuno seja menor do que do íleo do ponto de vista funcional. Serão necessários outros estudos para esclarecer esta possibilidade. A desnervação mientérica provocou aumento da área das camadas mucosa e muscular, bem como do diâmetro do jejuno. Trata-se, portanto, de uma acentuação da resposta hiperplásica da mucosa jejunal como conseqüência da ressecção intestinal, resposta semelhante à da parede ileal que obtivemos anteriormente em condições experimentais similare ${ }^{9}$. Podemos concluir que o modelo experimental de desnervação jejunal associada à ressecção intestinal extensa mostrou-se eficaz em provocar uma resposta hiperplásica da mucosa que aumentou a superfície de absorção e peso dos animais. Em revisão recente publicada a respeito das opções cirúrgicas para a síndrome do intestino curto ${ }^{12}$ os autores mencionam que todos os métodos atualmente disponíveis têm altas taxas de insucesso e grande número de complicações. O método de desnervação que estamos investigando, constitui em uma promissora esperança neste sentido, caso aprimorado em futuros estudos.

\section{Conclusões}

1. A desnervação mientérica provocou aumento da área das camadas mucosa e muscular, bem como do diâmetro do jejuno.

2. A desnervação mientérica do jejuno associada à ressecção intestinal extensa acarreta melhora do ganho ponderal dos animais.

\section{Referências}

1. Figueroa-Colon R, Harris PR, Birdsong E, Franklin FA, Georgeson KE. Impact of the intestinal lenghtening on the nutricional outcome for children with the short bowel syndrome. J Pediatr Surg. 1996; 31(7): 912-6.

2. Saday C, Mir E. A surgical model to increase the intestinal absortive surface: Intestinal lengthening and growing neo mucosa in the same approach. J Surg Res. 1996; 62: 184-91.

3. Bianchi A. Longitudinal intestinal lengthening and tailoring: results in 20 children. J R Soc Med. 1997; 90(8): 429-32.

4. Thompson JS. Edgar J. Poth Memorial Lecture. Surgical aspects of short-bowel syndrome. Am J Surg. 1995, 170(6): 532-6.

5. Pentillä A. Histochemical reactions of the enterochromaffin cells and the 5-hydroxytryptamine content of the mamalian duodenum. Acta Physiol Scand. 1966; 69:281-358.

6. Stollman NH, Neustoter BR, Rogers A. Short bowel syndrome: a review. Gastroenterologist. 1996; 4(2): 11828.

7. Aiken KD, Yu W, Wright JRJr. Roth KA. Adaptation of enteroendocrine cells in response to jejunal-ileal transposition in the rat. Gastroenterology. 1994; 106(6): 1576-83.

8. Sawchuk A, Goto S, Yount J, Grosfeld JA, Lohmuller J. Chemmically induced bowel denervation improves survival in short bowel syndrome. J Pediatr Surg. 1987; 22(6): 492-6.

9. Garcia SB, Kawasaky MC, Silva JCF, Garcia-Rodrigues AC, Bovo TJ, Iglesias AC, Zucoloto S. Intrinsic myenteric denervation: a new model to increase the intestinal absortive surface in short-bowel syndrome. J Surg Res. 1999; 85:200-3.

10. Senior PV, Prichett CJ, Sunter JP, Appleton DR, Watson AJ. A cytokinetic study of small-intestinal and colonic mucosa after resection of $70 \%$ of the small intestine. Cell Tissue Kinet. 1986; 19: 491-502. 
11. Santer RM, Baker DM. Enteric neuron numbers and sizes in Auerbach's plexus in the small and large intestine of adult and aged rats. J Auton Nerv Syst. 1988; 25: 59-67.
12. Vernon AH, Georgeson KE. Surgical options for short bowel syndrome. Semin Pediatr Surg. 2001; 10(2): 91-8.

\section{Correspondência:}

Sergio Brito Garcia

Av. Bandeirantes, 3900

14049-900 Ribeirão Preto - SP

sbgarcia@fmrp.usp.br
Conflito de interesse: nenhum Fonte de financiamento: nenhuma

Recebimento: 18/09/2005

Revisão: 10/10/2005

Aprovação: 04/11/2005

\section{Como citar este artigo:}

Carvalho CEV, Basile FVA, Vespúcio MVO, Iglesias ACG, Gava NF, Garcia SB. Efeitos da desnervação intrínseca do jejuno após enterectomia extensa na síndrome do intestino curto em ratos. Acta Cir Bras. [periódico na Internet] 2006 JanFev;21(1). Disponível em URL: http://www.scielo.br/acb

\section{AVISO AOS AUTORES}

\section{ESTILO VANCOUVER 2004: MUDANÇAS NAS REFERÊNCIAS}

As abreviaturas dos periódicos cumprem o INDEX MEDICUS.

ATENÇÃO: Após a última abreviatura do título do periódico (ou com título sem abreviatura) incluir um ponto final.

\section{Acessar www.icmje.org}

Consultar exemplar recente da Acta Cirúrgica Brasileira para servir de modelo. 